\title{
A NEW INFORMATION INEQUALITY AND ITS APPLICATION IN ESTABLISHING RELATION AMONG VARIOUS f-DIVERGENCE MEASURES
}

\author{
K.C.JAIN AND RAM NARESH SARASWAT
}

\begin{abstract}
An Information inequality by using convexity arguments and Jensen inequality is established in terms of Csiszar f-divergence measures. This inequality is applied in comparing particular divergences which play a fundamental role in Information theory, such as Kullback-Leibler distance, Hellinger discrimination, Chi-square distance, Jdivergences and others.
\end{abstract}

Mathematics Subject Classification 2000: 62B-10, 94A-17, 26D15

Additional Key Words and Phrases: - f-divergence measure, Chi-square divergence, Kullback-Leibler distance, Hellinger discrimination, J-divergence, Relative J-divergence, Jenson-Shannon's divergence, Triangular discrimination etc.

\section{INTRODUCTION}

Let

$\Gamma_{n}=\left\{P=\left(p_{1}, p_{2}, \ldots \ldots . p_{n}\right) \mid p_{i} \geq 0, \sum_{i=1}^{n} p_{i}=1\right\}, n \geq 2$

be the set of all complete finite discrete probability distributions. There are many information and divergence measures exists in the literature on information theory and statistics. Csiszar [4] \& [5] introduced a generalized measure of information using fdivergence measure

$$
I_{f}(P, Q)=\sum_{i=1}^{n} q_{i} f\left(\frac{p_{i}}{q_{i}}\right)
$$

where $f: \mathbf{R}_{+} \rightarrow \mathbf{R}_{+}$is a convex function and $P, Q \in \Gamma_{n}$. 
As in Csiszar [5], we have interpret undefined expressions by $f(0)=\lim _{t \rightarrow 0^{+}} f(t), 0 f\left(\frac{0}{0}\right)=0 \quad, \quad 0 f\left(\frac{a}{0}\right)=\lim _{\varepsilon \rightarrow 0^{+}} \varepsilon f\left(\frac{a}{\varepsilon}\right)=a \lim _{t \rightarrow \infty} \frac{f(t)}{t}, a>0$

Here we shall give some example of divergence measures in the category of Csiszar's fdivergence measure.

- Kullback-Leibler distance [10]:-

$$
D(P, Q)=\sum_{i=1}^{n} p_{i} \log \frac{p_{i}}{q_{i}}
$$

- Relative information of type s [14]:-

$$
D_{s}(P, Q)=[s(s-1)]^{-1}\left[\sum_{i=1}^{n} p_{i}^{s} q_{i}^{1-s}-1\right], s \neq 0,1
$$

which is one parametric generalization of measure (1.2) for $\mathrm{s}=0$ and $\mathrm{s}=1$ it reduces to measure $\mathrm{D}(\mathrm{P}, \mathrm{Q})$ and $\mathrm{D}(\mathrm{Q}, \mathrm{P})$ respectively given by $(1.2)$

- Chi-square Divergence [11]:-

$$
\chi^{2}(P, Q)=\sum_{i=1}^{n} \frac{\left(p_{i}-q_{i}\right)^{2}}{q_{i}}=\sum_{i=1}^{n} \frac{p_{i}^{2}}{q_{i}}-1
$$

- Relative Jensen-Shannon divergence [15]:-

$$
F(P, Q)=\sum_{i=1}^{n} p_{i} \log \left(\frac{2 p_{i}}{p_{i}+q_{i}}\right)
$$

- Relative arithmetic-geometric divergence [3]:-

$$
G(P, Q)=\sum_{i=1}^{n}\left(\frac{p_{i}+q_{i}}{2}\right) \log \left(\frac{p_{i}+q_{i}}{2 p_{i}}\right)
$$

- Unified relative Jensen-Shannon and Arithmetic-Geometric Divergence of type s [14] :- $\quad F G_{s}(P, Q)=[s(s-1)]^{-1}\left[\sum_{i=1}^{n} p_{i}\left(\frac{p_{i}+q_{i}}{2 q_{i}}\right)^{s}-1\right], s \neq 0,1$ 
which is one parametric generalization for the measures (1.5) \& (1.6) for $s=0$ and $s=1$ it reduces to (1.5) \& (1.6) respectively.

- Hellinger Discrimination [1]:-

$$
h(P, Q)=1-B(P, Q)=\frac{1}{2} \sum_{i=1}^{n}\left(\sqrt{p_{i}}-\sqrt{q_{i}}\right)^{2}
$$

where $B(P, Q)=\sum_{i=1}^{n} \sqrt{p_{i} q_{i}} \quad$ is known as Bhattacharya divergence measure $[2]$

- Triangular Discrimination [6]:-

$$
\Delta(P, Q)=2[1-W(P, Q)]=\sum_{i=1}^{n} \frac{\left(p_{i}-q_{i}\right)^{2}}{p_{i}+q_{i}}
$$

where $\quad W(P, Q)=\sum_{i=1}^{n} \frac{2 p_{i} q_{i}}{p_{i}+q_{i}} \quad$ is known as harmonic mean divergence measure

- Chi-square Divergence [8]:-

$$
\psi(P, Q)=\chi^{2}(P, Q)+\chi^{2}(Q, P)=\sum_{i=1}^{n} \frac{\left(p_{i}-q_{i}\right)^{2}\left(p_{i}+q_{i}\right)}{p_{i} q_{i}}
$$

- J-Divergence measure [5]:-

$$
\begin{aligned}
& J(P, Q)=\sum_{i=1}^{n}\left(p_{i}-q_{i}\right) \log \frac{p_{i}}{q_{i}} \\
& =D(P, Q)+D(P, Q)
\end{aligned}
$$


- Relative J-Divergence measure [12]:-

$$
J_{R}(P, Q)=\sum_{i=1}^{n}\left(p_{i}-q_{i}\right) \log \frac{p_{i}+q_{i}}{2 q_{i}}
$$

- Arithmetic-geometric divergence [13]: -

$$
T(P, Q)=\frac{1}{2}[G(P, Q)+G(Q, P)]=\sum_{i=1}^{n}\left(\frac{p_{i}+q_{i}}{2}\right) \log \left(\frac{p_{i}+q_{i}}{2 \sqrt{p_{i} q_{i}}}\right)
$$

- Symmetric Chi-square, Arithmetic and Geometric mean divergence measure [9]:-

$$
E_{1}^{*}(P, Q)=\sum_{i=1}^{n} \frac{\left(p_{i}-q_{i}\right)^{2}}{\sqrt{p_{i} q_{i}}}
$$

- Jensen-Shannon divergence measure $[3,16]:-$

$$
I(P, Q)=\frac{1}{2}[F(P, Q)+F(Q, P)]=\frac{1}{2}\left[\sum_{i=1}^{n} p_{i} \log \left(\frac{2 p_{i}}{p_{i}+q_{i}}\right)+\sum_{i=1}^{n} q_{i} \log \left(\frac{2 q_{i}}{p_{i}+q_{i}}\right)\right]
$$

2. New information inequality: -We prove the following theorem regarding information inequality using Jensen inequality.

Theorem 2.1:-Let $f: \mathbf{R}_{+} \rightarrow \mathbf{R}_{+}$be the differentiable convex function and normalized i.e. $f(1)=0$. Then for all $P, Q \in \Gamma_{n}$ we have the following inequality

$$
\begin{gathered}
\sum_{i=1}^{n} q_{i} f\left(\frac{p_{i}+q_{i}}{2 q_{i}}\right) \leq \frac{1}{2} \sum_{i=1}^{n} q_{i} f\left(\frac{p_{i}}{q_{i}}\right) \\
S_{f}(P, Q) \leq \frac{1}{2} I_{f}(P, Q) \\
20
\end{gathered}
$$




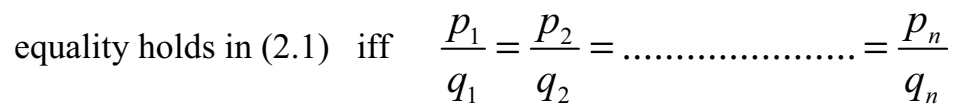

where $\quad S_{f}(P, Q)=\sum_{i=1}^{n} q_{i} f\left(\frac{p_{i}+q_{i}}{2 q_{i}}\right)$ we call a new f-divergence measure.

and $\quad I_{f}(P, Q)=\sum_{i=1}^{n} q_{i} f\left(\frac{p_{i}}{q_{i}}\right) \quad$ same as (1.1)

Proof:-Let $f: I \subset \mathbf{R} \rightarrow \mathbf{R}$ be a differentiable convex function on the interval I, $t_{i} \in \stackrel{0}{I}(\stackrel{0}{I}$ is interior of $\mathrm{I})$.

Let $\lambda=\left(\lambda_{1}, \lambda_{2}, \ldots \ldots . . \lambda_{n}\right) \in \Gamma_{n}$, then the Jensen inequality is

$$
\begin{aligned}
& f\left(\sum_{i=1}^{n} \lambda_{i} t_{i}\right) \leq \sum_{i=1}^{n} \lambda_{i} f\left(t_{i}\right) \\
& \text { If } \lambda_{1}=\lambda_{2}=\frac{1}{2}, \lambda_{3}=\lambda_{4}=\ldots \ldots \ldots \ldots=\lambda_{n}=0 \text {, then equation (2.2) reduced to } \\
& \qquad f\left(\frac{t_{1}+t_{2}}{2}\right) \leq \frac{1}{2}\left[f\left(t_{1}\right)+f\left(t_{2}\right)\right]
\end{aligned}
$$

Putting $t_{1}=t, t_{2}=1$ then

$$
\begin{aligned}
f\left(\frac{t+1}{2}\right) & \leq \frac{1}{2}[f(t)+f(1)] \\
f\left(\frac{t+1}{2}\right) & \leq \frac{1}{2} f(t), \text { since } f(1)=0
\end{aligned}
$$

Putting $t=\frac{p_{i}}{q_{i}}$ in equation (2.5) 


$$
f\left(\frac{p_{i}+q_{i}}{2 q_{i}}\right) \leq \frac{1}{2} f\left(\frac{p_{i}}{q_{i}}\right)
$$

Multiplying by $q_{i}$ and taking summation both side

$$
\begin{gathered}
\sum_{i=1}^{n} q_{i} f\left(\frac{p_{i}+q_{i}}{2 q_{i}}\right) \leq \frac{1}{2} \sum_{i=1}^{n} q_{i} f\left(\frac{p_{i}}{q_{i}}\right) \\
S_{f}(P, Q) \leq \frac{1}{2} I_{f}(P, Q)
\end{gathered}
$$

equation (2.1) is a generalized inequality relating the Csiszar's f-divergence measure and a new f-divergence measure.

\section{Applications for some particular f-divergences:-}

In this section we will show relationship in the form of inequality using the results of theorem (2.1) among the various known measures such as Kullback-Leibler, Hellinger, Chi-square, and J-divergence etc.

Result: 3.1- Let $P, Q \in \Gamma_{n}$, then we have the following relations

$$
F(P, Q) \leq \frac{1}{2} D(P, Q)
$$

$$
F(Q, P) \leq \frac{1}{2} D(Q, P)
$$

where $F(P, Q)$ and $D(P, Q)$ are Relative Jensen Shannon divergence measure and Kullback-Leibler divergence measure give in (1.5) and (1.2) respectively..

Proof: - Considering the mapping $f:(0, \infty) \rightarrow \mathbf{R}$

$$
f(t)=-\log t, f^{\prime \prime}(t)=\frac{1}{t^{2}}>0, \forall t>0
$$


A NEW INFORMATION INEQUALITY AND ITS APPLICATION IN ...

$f^{\prime \prime}(t) \geq 0$ and $f(1)=0$, So function $\mathrm{f}$ is convex and normalized.

then

$$
S_{f}(P, Q)=\sum_{i=1}^{n} q_{i} f\left(\frac{p_{i}+q_{i}}{2 q_{i}}\right)=\sum_{i=1}^{n} q_{i} \log \left(\frac{2 q_{i}}{p_{i}+q_{i}}\right)=F(Q, P)
$$

Interchange by $P \rightarrow Q$

$S_{f}(Q, P)=\sum_{i=1}^{n} p_{i} f\left(\frac{p_{i}+q_{i}}{2 p_{i}}\right)=\sum_{i=1}^{n} p_{i} \log \left(\frac{2 p_{i}}{p_{i}+q_{i}}\right)=F(P, Q)$

$I_{f}(P, Q)=\sum_{i=1}^{n} q_{i} \log \frac{q_{i}}{p_{i}}=D(Q, P)$

Interchange by $P \rightarrow Q$

$$
I_{f}(Q, P)=\sum_{i=1}^{n} p_{i} \log \frac{p_{i}}{q_{i}}=D(P, Q)
$$

equation (2.1) using (3.3), (3.4), (3.5) \& (3.6) gives the results (3.1) \& (3.2)

Results: 3.2- Let $P, Q \in \Gamma_{n}$, then we have the following relation

$$
G(Q, P) \leq \frac{1}{2} D(Q, P)
$$


where $G(Q, P)$ and $D(Q, P)$ are relative Arithematic-Geometric and Kullback-Leibler divergence measure give in (1.6) and (1.2) respectively.

Proof: - Considering mapping $f:(0, \infty) \rightarrow \mathbf{R}$

$$
\begin{aligned}
& f(t)=t \log t, f^{\prime \prime}(t)=\frac{1}{t}, \forall t>0 \\
& f^{\prime \prime}(t) \geq 0 \text { and } f(1)=0, \text { So function } \mathrm{f} \text { is convex and normalized. }
\end{aligned}
$$

$$
S_{f}(P, Q)=\sum_{i=1}^{n} q_{i} \log \left(\frac{p_{i}+q_{i}}{2 q_{i}}\right)=G(Q, P)
$$

and

$$
I_{f}(P, Q)=\sum_{i=1}^{n} q_{i}\left[\frac{p_{i}}{q_{i}} \log \frac{p_{i}}{q_{i}}\right]=\sum_{i=1}^{n} p_{i} \log \frac{p_{i}}{q_{i}}=D(P, Q)
$$

equation (2.1) using (3.8) \& (3.9) gives the result (3.7) .

Results: 3.3- Let $P, Q \in \Gamma_{n}$, then we have the following relations

$$
\begin{aligned}
& J_{R}(P, Q) \leq J(P, Q) \\
& I(P, Q)+T(P, Q) \leq \frac{1}{2} J(P, Q)
\end{aligned}
$$

where $J(P, Q), J_{R}(P, Q), T(P, Q)$ and $I(P, Q)$ are J-divergence, Relative Jdivergence, Arithmetic-geometric divergence, and Jensen-Shannon divergence measure gives in (1.11),(1.12), (1.13) and (1.16) respectively. 
A NEW INFORMATION INEQUALITY AND ITS APPLICATION IN ...

Proof: - Considering the mapping $f:(0, \infty) \rightarrow \mathbf{R}$

$f(t)=(t-1) \log t, f^{\prime \prime}(t)=\frac{t+1}{t^{2}}, \forall t>0$

$f^{\prime \prime}(t) \geq 0$ and $f(1)=0$, So function $\mathrm{f}$ is convex and normalized.

then

$$
\begin{gathered}
S_{f}(P, Q)=\sum_{i=1}^{n} q_{i} f\left(\frac{p_{i}+q_{i}}{2 q_{i}}\right)=\frac{1}{2} \sum_{i=1}^{n}\left(p_{i}-q_{i}\right) \log \left(\frac{p_{i}+q_{i}}{2 q_{i}}\right)=\frac{1}{2} J_{R}(P, Q) \\
I_{f}(P, Q)=\sum_{i=1}^{n} q_{i} f\left(\frac{p_{i}}{q_{i}}\right)=\sum_{i=1}^{n}\left(p_{i}-q_{i}\right) \log \left(\frac{p_{i}}{q_{i}}\right)=J(P, Q)
\end{gathered}
$$

equation (2.1) using (3.12) \& (3.13) gives (3.10)

Now again we get

$$
\begin{aligned}
& S_{f}(P, Q)=\frac{1}{2} \sum_{i=1}^{n}\left[p_{i} \log \left(\frac{p_{i}+q_{i}}{2 q_{i}}\right)-q_{i} \log \left(\frac{p_{i}+q_{i}}{2 q_{i}}\right)\right] \\
& \Rightarrow \\
& =\frac{1}{2} \sum_{i=1}^{n}\left[p_{i} \log \left(\frac{2 p_{i}}{p_{i}+q_{i}}\right)+q_{i} \log \left(\frac{2 q_{i}}{p_{i}+q_{i}}\right)\right]+\frac{1}{2} \sum_{i=1}^{n} p_{i} \log \left(\frac{p_{i}+q_{i}}{2 q_{i}} \cdot \frac{p_{i}+q_{i}}{2 p_{i}}\right) \\
& \Rightarrow S_{f}(P, Q)=I(P, Q)+\sum_{i=1}^{n} p_{i} \log \left(\frac{p_{i}+q_{i}}{2 \sqrt{p_{i} q_{i}}}\right)
\end{aligned}
$$

using equation (2.1), (3.13) \& (3.14), we get

$$
I(P, Q)+\sum_{i=1}^{n} p_{i} \log \left(\frac{p_{i}+q_{i}}{2 \sqrt{p_{i} q_{i}}}\right) \leq \frac{1}{2} J(P, Q)
$$

Interchanging P \& Q we get 


$$
I(Q, P)+\sum_{i=1}^{n} q_{i} \log \left(\frac{p_{i}+q_{i}}{2 \sqrt{p_{i} q_{i}}}\right) \leq \frac{1}{2} J(Q, P)
$$

adding inequalities (3.15) \& (3.16) both side

$$
\begin{aligned}
& I(P, Q)+I(Q, P)+\sum_{i=1}^{n}\left(p_{i}+q_{i}\right) \log \left(\frac{p_{i}+q_{i}}{2 \sqrt{p_{i} q_{i}}}\right) \leq \frac{1}{2} J(P, Q)+\frac{1}{2} J(Q, P) \\
& 2 I(P, Q)+2 T(P, Q) \leq \sum_{i=1}^{n}\left(p_{i}-q_{i}\right) \log \frac{p_{i}}{q_{i}} \\
& \because \quad I(P, Q)=I(Q, P) \\
& 2 I(P, Q)+2 T(P, Q) \leq J(P, Q) \\
& I(P, Q)+T(P, Q) \leq \frac{1}{2} J(P, Q) \text { which is required result, }
\end{aligned}
$$

Note: - From equation (3.11) we get

(a) $I(P, Q) \leq \frac{1}{2} J(P, Q)$

(b) $T(P, Q) \leq \frac{1}{2} J(P, Q)$, with equality if $P=Q$

Since $I(P, Q) \& T(P, Q)$ both are positive.

Results: 3.4- Let $P, Q \in \Gamma_{n}$, then we have the following relation

$$
\Delta(P, Q)<E_{1}^{*}(P, Q)
$$

where $\Delta(P, Q)$ and $E_{1}^{*}(P, Q)$ are Triangular discrimination and Symmetric Chisquare, Arithmetic and Geometric mean divergence measure give in (1.9) and (1.14) respectively. 
Proof: - Considering the mapping $f:(0, \infty) \rightarrow \mathbf{R}$

$$
f(t)=\frac{(t-1)^{2}}{\sqrt{t}}, f^{\prime \prime}(t)=\frac{3}{4} t^{-\frac{1}{2}}+\frac{3}{4} t^{-\frac{5}{2}}+\frac{1}{2} t^{-\frac{3}{2}}>0, \forall t>0
$$

$f^{\prime \prime}(t) \geq 0$ and $f(1)=0$, So function $\mathrm{f}$ is convex and normalized.

then

$$
S_{f}(P, Q)=\sum_{i=1}^{n} q_{i} f\left(\frac{p_{i}+q_{i}}{2 q_{i}}\right)=\sum_{i=1}^{n} \frac{\left(p_{i}-q_{i}\right)^{2}}{\sqrt{2 q_{i}\left(p_{i}+q_{i}\right)}}
$$

$$
I_{f}(P, Q)=\sum_{i=1}^{n} q_{i} f\left(\frac{p_{i}}{q_{i}}\right)=\sum_{i=1}^{n} \frac{\left(p_{i}-q_{i}\right)^{2}}{\sqrt{p_{i} q_{i}}}=E_{1}^{*}(P, Q)
$$

from equation $(2.1),(3.18) \&(3.19)$

$$
\sum_{i=1}^{n} \frac{\left(p_{i}-q_{i}\right)^{2}}{\sqrt{2 q_{i}\left(p_{i}+q_{i}\right)}} \leq \frac{1}{2} E_{1}^{*}(P, Q)
$$$$
2 \sum_{i=1}^{n} \frac{\left(p_{i}-q_{i}\right)^{2}}{\sqrt{2 q_{i}\left(p_{i}+q_{i}\right)}} \leq E_{1}^{*}(P, Q)
$$$$
\Delta(P, Q)=\sum_{i=1}^{n} \frac{\left(p_{i}-q_{i}\right)^{2}}{\left(p_{i}+q_{i}\right)}<\sqrt{2} \sum_{i=1}^{n} \frac{\left(p_{i}-q_{i}\right)^{2}}{\sqrt{\left(p_{i}^{2}+q_{i}^{2}+2 p_{i} q_{i}\right)}}<\sqrt{2} \sum_{i=1}^{n} \frac{\left(p_{i}-q_{i}\right)^{2}}{\sqrt{q_{i}\left(p_{i}+q_{i}\right)}}<E_{1}^{*}(P, Q)
$$

$\Delta(P, Q)<E_{1}^{*}(P, Q)$ hence the relation (3.17) is proved.

Results: 3.5- Let $P, Q \in \Gamma_{n}$, then we have the following relation 
$\frac{1}{2} \chi^{2}(P, Q)+\Delta(P, Q) \leq \Psi(P, Q)$

where $\chi^{2}(P, Q), \Delta(P, Q)$ and $\Psi(P, Q)$ are Chi-square divergence,

Triangular discrimination and Symmetric Chi-square divergence measure give in (1.4), (1.9) and (1.10) respectively.

Proof: - Considering the mapping $f:(0, \infty) \rightarrow \mathbf{R}$

$$
\begin{aligned}
& f(t)=\frac{(t-1)^{2}(t+1)}{t} f^{\prime \prime}(t)=2+\frac{2}{t^{3}}, \forall t>0 \\
& f^{\prime \prime}(t) \geq 0 \text { and } f(1)=0, \text { So function } \mathrm{f} \text { is convex and normalized. } \\
& S_{f}(P, Q)=\sum_{i=1}^{n} q_{i} f\left(\frac{p_{i}+q_{i}}{2 q_{i}}\right)=\sum_{i=1}^{n} \frac{\left(p_{i}-q_{i}\right)^{2}\left(p_{i}+3 q_{i}\right)}{4 q_{i}\left(p_{i}+q_{i}\right)} \\
& =\frac{1}{4} \sum_{i=1}^{n} \frac{\left(p_{i}-q_{i}\right)^{2}}{q_{i}}+\frac{1}{2} \sum_{i=1}^{n} \frac{\left(p_{i}-q_{i}\right)^{2}}{\left(p_{i}+q_{i}\right)} \\
& S_{f}(P, Q)=\sum_{i=1}^{n} q_{i} f\left(\frac{p_{i}+q_{i}}{2 q_{i}}\right)=\frac{1}{4} \chi^{2}(P, Q)+\frac{1}{2} \Delta(P, Q) \\
& I_{f}(P, Q)=\sum_{i=1}^{n} q_{i} f\left(\frac{p_{i}}{q_{i}}\right)=\Psi(P, Q)
\end{aligned}
$$

equation (2.1) using (3.21) \& (3.22) gives the result (3.20) .

Results: 3.6- Let $P, Q \in \Gamma_{n}$, then we have the following relation

$$
F G_{s}(Q, P) \leq \frac{1}{2}^{2} K_{s}(P, Q)
$$


where $F G_{s}(P, Q)$ and $K_{s}(P, Q)$ Unified relative Jensen-Shannon and ArithmeticGeometric Divergence of type s and Relative information of type s give in (1.7) and (1.3) respectively.

Proof: - Considering the mapping $f:(0, \infty) \rightarrow \mathbf{R}$

$$
f_{s}(t)= \begin{cases}{[s(s-1)]^{-1}\left[t^{s}-1\right],} & s \neq 0,1 \\ -\log t & \text { if } s=0 \\ t \log t & \text { if } s=1\end{cases}
$$

$$
\begin{aligned}
& f^{\prime \prime}(t)=t^{s-2}>0, \forall t>0 \\
& f^{\prime \prime}(t) \geq 0 \text { and } f(1)=0, \text { So function } \mathrm{f} \text { is convex and normalized. }
\end{aligned}
$$

then

$$
S_{f}(P, Q)=\sum_{i=1}^{n} q_{i} f\left(\frac{p_{i}+q_{i}}{2 q_{i}}\right)=[s(s-1)]^{-1}\left[\sum_{i=1}^{n} q_{i}\left(\frac{p_{i}+q_{i}}{2 q_{i}}\right)^{s}-1\right]=F G_{s}(Q, P)
$$

$$
I_{f}(P, Q)=\frac{1}{2} \sum_{i=1}^{n} q_{i} f\left(\frac{p_{i}}{q_{i}}\right)=[s(s-1)]^{-1}\left[\sum_{i=1}^{n} p_{i}^{s} q_{i}^{1-s}-1\right]={ }^{2} K_{s}(P, Q)
$$

equation (2.1) using (3.24), (3.25) \& (3.26) gives the result (3.23)

\section{Particular Cases of Result 3.6:-}

(i) If $\mathrm{s}=0,1$ then we have proved in equation (3.1) \& (3.7) of results $3.1 \& 3.2$ respectively.

(ii) If $s=-1$ and let $P, Q \in \Gamma_{n}$, then we have the following relation

$$
\Delta(P, Q) \leq \chi^{2}(Q, P)
$$


Proof: - Put $s=-1$ in equation (3.23), then

$$
F G_{-1}(Q, P) \leq \frac{1}{2}^{2} K_{-1}(P, Q)
$$

where

$$
\begin{aligned}
& F G_{-1}(Q, P)=\frac{1}{2}\left[\sum_{i=1}^{n} \frac{2 q_{i}^{2}}{p_{i}+q_{i}}-1\right]=\frac{1}{2}\left[\sum_{i=1}^{n}\left(2 q_{i}-\frac{2 p_{i} q_{i}}{p_{i}+q_{i}}\right)-1\right]=\frac{1}{2}\left[2-\sum_{i=1}^{n} \frac{2 p_{i} q_{i}}{p_{i}+q_{i}}-1\right] \\
& F G_{-1}(Q, P)=\frac{1}{2}\left[1-\sum_{i=1}^{n} \frac{2 p_{i} q_{i}}{p_{i}+q_{i}}\right] \\
& F G_{-1}(Q, P)=\frac{1}{2}[1-W(P, Q)] \\
& F G_{-1}(Q, P)=\frac{1}{4} \Delta(P, Q)[\text { using (1.9) and (3.25)] }
\end{aligned}
$$

and

${ }^{2} K_{-1}(P, Q)=\frac{1}{2} \chi^{2}(Q, P) \quad[$ using (1.4) and (3.26) $]$

using equation (3.28), (3.29) \& (3.30) gives the result (3.27)

(iii) If $s=\frac{1}{2}$ and let $P, Q \in \Gamma_{n}$, then we have the following relation

$$
4\left[1-B\left(\frac{P+Q}{2}, Q\right)\right] \leq h(P, Q)
$$

Proof: - Put $s=\frac{1}{2}$ in equation (3.23), then

$$
F G_{1 / 2}(Q, P) \leq \frac{1}{2}^{2} K_{1 / 2}(P, Q)
$$




\section{A NEW INFORMATION INEQUALITY AND ITS APPLICATION IN ...}

where

$$
\begin{aligned}
& F_{G_{\frac{1}{2}}}(Q, P)=-4\left[\sum_{i=1}^{n} q_{i}\left(\frac{p_{i}+q_{i}}{2 q_{i}}\right)^{\frac{1}{2}}-1\right]=4\left[1-\sum_{i=1}^{n} \sqrt{\frac{p_{i} q_{i}+q_{i}^{2}}{2}}\right]=4\left[1-B\left(\frac{P+Q}{2}, Q\right)\right] \\
& \Rightarrow F_{G_{\frac{1}{2}}}(Q, P)=4\left[1-B\left(\frac{P+Q}{2}, Q\right)\right] \quad[\text { using (1.8) and (3.25)] }
\end{aligned}
$$

and

$$
\begin{aligned}
& { }_{\frac{1}{2}}^{2} K_{1}(P, Q)=-4\left[\sum_{i=1}^{n} \sqrt{p_{i} q_{i}}-1\right]=4\left[1-\sum_{i=1}^{n} \sqrt{p_{i} q_{i}}\right]=4[1-B(P, Q)]=2 h(P, Q) \\
& { }^{2} K_{\frac{1}{2}}(P, Q)=2 h(P, Q) \quad[\operatorname{using}(1.8) \text { and }(3.26)]
\end{aligned}
$$

using equation (3.32), (3.33) \& (3.34) gives the result (3.31)

\section{REFERENCES}

[1] Beran R., Minimum Hellinger distance estimates for parametric models Ann.Statist.5 (1977), 445-463

[2] Bhattacharya A., Some analogues to amount of information and their uses in statistical estimation, Sankhya8 (1946) 1-14

[3] Burbea J., and C.R.Rao, On Convexity of Some Divergence measures based on entropy functions,IEEE Transe.on.inform.theory ,IT-28 (1982),489-495

[4] Csiszar I.Information measure, A critical servey.Trans. $7^{\text {th }}$ prague conf.on info. Th. Statist. Decius. Funct, Random Processes and $8^{\text {th }}$ European meeting of statist Volume B.Acadmia Prague, 1978, PP-73-86

[5] Csiszar I.,Information-type measures of difference of probability functions and indirect observations. studia Sci.Math.hunger.2(1961).299-318

[6] Dragomir S.S., Bounds of f-divergences under likelihood Ratio Constraints No.3, 205-223, 48(2003)

[7] Dragomir S.S., V.gluscevic and C.E.M. pearce, approximation for the csiszar f -divergence via mid-point inequalities, in inequality theory and applications-Y.J.Cho,J.K.Kim and S.S.dragomir(Eds),nova science publishers, inc., Huntington, new York, vol1, 2001, pp.139-154. 


\section{K.C.JAIN, R. N. SARASWAT}

[8] Dragomir S.S., J.Sunde and C.Buse, New Inequalities for Jeffreys Divergence measure,Tamusi Oxford Journal of Mathematical Sciences,16(2)(2000),295-309

[9] Jain K.C. and A. Srivastava, On Symmetric Information Divergence Measures of Csiszar's f-Divergence Class, Journal of Applied Mathematics, Statistics and Informatics (JAMSI),3(1)(2007),85-102

[10] Kullback S., R.A Leibler:On information and sufficiency.Ann.Nath.Statistics 22(1951),79-86

[11] Pearson K., On the criterion that a give system of deviations from the probable in the case of correlated system of variables in such that it can be reasonable supposed to have arisen from random sampling,Phil. Mag., 50(1900), 157-172.

[12] Taneja I.J. and P.Kumar, Relative information of type s ,Csiszar f-divergence and Information inequalities, Information Sciences,166 (1-6)(2004),105-125 Also in: http:// rgmia.vu.edu.au, RGMIA Research Report Collection.6(3)(2003). Article12

[13] Taneja I.J., New Developments in generalized information measures, Chapter in: Advances in imaging and Electron Physics, Ed. P.W. Hawkes 91 (1995),37-135.

[14] Taneja I.J., Pranesh Kumar, Generalized non-symmetric divergence measures and inequalities.

[15] Topse F., Some inequalities for information divergence and related measures of discrimination.Res.Coll.RGMIA2 (1999), 85-98.

[16] Sibson R., Information Radius,Z,Wahrs.undverw.geb.(14)(1969),149-160

K. C. Jain

Department of Mathematics,

Malaviya National Institute of Technology,

Jaipur (Rajasthan) -302017, INDIA

E-mail: jainkc.2003@gmail.com

Ram Naresh Saraswat

Department of Mathematics,

Malaviya National Institute of Technology,

Jaipur (Rajasthan) -302017, INDIA

E-mail: sarswatrn@gmail.com

Received October 2011 